\title{
Reversible endotracheal tube obstruction caused by meconium acting as a check valve
}

\author{
Wonjung Hwang, Jieun Hwang, and Joonpyo Jeon
}

Department of Anesthesiology and Pain Medicine, Seoul St. Mary's Hospital, College of Medicine, The Catholic University of Korea, Seoul, Korea

Endotracheal intubation is necessary in almost all cases of general anesthesia to maintain the airway. The endotracheal tube (ETT) sometimes causes airway obstruction secondary to secretions or other intraluminal materials, and it may cause hypoxemia and fatal outcomes [1,2]. We herein describe a case of a reversible ETT obstruction caused by meconium acting as a check valve, which was mistaken initially to be leakage from the anesthetic machine.

The patient was a 1-day-old male infant (height, $43 \mathrm{~cm}$; weight, $1.7 \mathrm{~kg}$ ). He was born by cesarean delivery at gestational age $34^{+3}$ weeks due to an omphalocele. The ruptured sac of the omphalocele (approximately $5 \mathrm{~cm}$ in diameter) was found at birth, and the emergency operation was planned. Before the surgery, he was already intubated because of meconium aspiration.

In the operating room, initial blood pressure and heart rate were 52/32 $\mathrm{mmHg}$ and $160 / \mathrm{min}$, respectively, and $\mathrm{SpO}_{2}$ was $98 \%$. A $3.0 \mathrm{~mm}$ uncuffed ETT had been placed at a depth of $8.5 \mathrm{~cm}$, and a small amount of gas leakage was confirmed at the peak inflation pressure of $20 \mathrm{cmH}_{2} \mathrm{O}$. Both lungs were clear by auscultation, and no secretions drained from the ETT upon suction. The patient underwent pressure-controlled ventilation using the following settings: inspiratory pressure, $15 \mathrm{cmH}_{2} \mathrm{O}$; respiratory rate, $30 / \mathrm{min}$; I : E ratio, $1: 2$. The tidal volume and end-tidal $\mathrm{CO}_{2}\left(\mathrm{ETCO}_{2}\right)$ were maintained at approximately $18-20 \mathrm{ml}$ and $32-35 \mathrm{mmHg}$, respectively. $\mathrm{SpO}_{2}$ was maintained at $>95 \%$ during the operation.

The surgeon repaired the entire intestine and began to close the abdominal cavity 3 hours after the surgical incision. Cap- nography showed an obstructive pattern, and $\mathrm{ETCO}_{2}$ increased slightly to $38-40 \mathrm{mmHg}$ with no change in ventilator settings. We suctioned the ETT again using a 6-Fr suction tip, and there was no resistance or secretion.

Upon completion of the abdominal closure, the surgeon decided to place an internal jugular venous catheter for postoperative care. The ventilator leakage alarm sounded during venous cut-down. The tidal volume decreased from 20 to $5-7 \mathrm{ml}$, and ETCO $_{2}$ decreased to $<10 \mathrm{mmHg}$. The capnography curve exhibited a blunt concave slope during the expiratory phase. The ETT was confirmed to be positioned correctly, and there was little gas leakage around the ETT. A full examination of the anesthetic machine and breathing circuit, including capnography, revealed no abnormalities, but the $\mathrm{SpO}_{2}$ had decreased to $88 \%$. Upon switching to manual ventilation during examination, we encountered resistance during the inspiratory phase. Chest auscultation revealed inspiratory rales and faint expiratory wheezing in both lung fields. The ETT suction was repeated, but no secretion was seen. The ETT was removed, and we reintubated with another $3.0 \mathrm{~mm}$ uncuffed ETT under direct laryngoscopy. The tidal volume was $20-25 \mathrm{ml}$ at an inspiratory pressure of 15 $\mathrm{cmH}_{2} \mathrm{O}$, and capnography showed a normal plot. The $\mathrm{ETCO}_{2}$ measured immediately after reintubation was $58 \mathrm{mmHg}$ and decreased to 33-35 mmHg after hyperventilation for 3 minutes. Inspection of the removed ETT revealed thick meconium approximately $2 \mathrm{~cm}$ long plugging the distal lumen of the ETT. Additionally, an approximately $0.3 \mathrm{~cm}$-long inspissated meconium was dangling from the superior portion of the inner wall

Corresponding author: Joonpyo Jeon, M.D., Ph.D., Department of Anesthesiology and Pain Medicine, The Catholic University of Korea, Seoul St. Mary's Hospital, 222, Banpo-daero, Seocho-gu, Seoul 137-701, Korea. Tel: 82-2-2258-6152, Fax: 82-2-537-1951, E-mail: anejjp@catholic.ac.kr (c) This is an open-access article distributed under the terms of the Creative Commons Attribution Non-Commercial License (http:// creativecommons.org/licenses/by-nc/3.0/), which permits unrestricted non-commercial use, distribution, and reproduction in any medium, provided the original work is properly cited. 


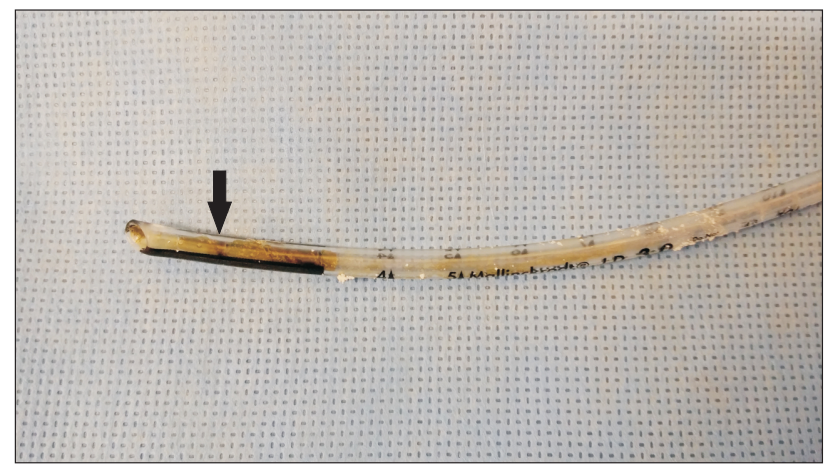

Fig. 1. Side view of the removed endotracheal tube showing obstruction of the distal lumen by meconium. The arrow indicates the dangling meconium, which acted as a check valve.

\section{of the ETT (Fig. 1).}

ETT obstruction due to bronchial secretions is usually suspected by abnormally high peak inspiratory pressures, a significant difference between the measured inhaled and exhaled tidal volumes, decreased lung compliance, increased resistance to inflation, and an obstructive pattern on capnography [3]. In the present case, the peak inspiratory pressure was constant because the pressure-controlled mode was set. The concave capnography plot with a low expiratory tidal volume caused the ETT obstruction to appear as a leak in the anesthetic machine. We initially suspected the leak around the ETT, because the patient's head was rotated for preparation of venous catheterization and there was the possibility of movement of the ETT. We also suspected leakage of the breathing circuit according to the capnography results, because sidestream devices typically extract a sample of gas of 50-200 $\mathrm{ml} / \mathrm{min}$, and the sampling volume often exceeds the fresh gas flow and causes anesthetic machine leaks in small pediatrics [4]. However, no ETT movement or leakage was confirmed, and no leak occurred at the same ventilation settings for more than 3 hours. Because in our pursuit of the cause, we were concerned only with the leak, the obstruction was identified belatedly.

Based on the inspection of the first ETT, the proposed sequence of events is as follows. The dangling meconium might have allowed for inspiration because positive-pressure ventilation was administered. After the termination of inspiration, the dangling meconium might have obstructed the lumen of the ETT and acted as a one-way check valve, preventing minimal gas egress. As a result, the expiratory tidal volume might have decreased, and a flow sensor in the expiratory limb must have detected this change as a leak in the anesthetic machine. Use of the pressure-controlled mode interrupted the early diagnosis of airway obstruction, but fortunately prevented an excessive increase in the intrathoracic pressure in the lung without a way to relieve it.

We used a heated breathing circuit with a humidifier in this case. However, the humidified inspiratory gas did not entirely prevent accumulation of meconium in the ETT, despite the fact that it may have induced slow accumulation. It was possible that the ETT obstruction occurred by the upward movement of meconium in the lower airway, as indicated by the changing shape of the capnography curve upon closure of the abdominal cavity. Moreover, the suction catheter may not have been inserted to a sufficient depth to reach the meconium, or the suction catheter that fit the small lumen of the ETT was too thin to remove the thick meconium. More meconium accumulated with time, and the dangling meconium may have acted as a check valve.

In conclusion, anesthesiologists should consider the possibility of delayed airway obstruction in meconium-stained babies. Frequent and sufficient suction must be performed in these patients. Additionally, when the leakage alarm of the anesthetic machine sounds, we suggest that the anesthesiologists consider the possibility of the plugged material acting as a check valve.

\section{References}

1. Capra GG, Shah AN, Moore JD, Halsey WS, Lujan E. Silicone-based endotracheal tube causing airway obstruction and pneumothorax. Arch Otolaryngol Head Neck Surg 2012; 138: 588-91.

2. Lim HK, Lee MH, Shim HY, Byon HJ, Ahn HS. Complete obstruction of an endotracheal tube due to an unexpected blood clot in a patient with a hemo-pneumothorax after repositioning of the patient for lumbar spine surgery. Korean J Anesthesiol 2013; 64: 382-3.

3. Wilson AM, Gray DM, Thomas JG. Increases in endotracheal tube resistance are unpredictable relative to duration of intubation. Chest 2009; 136: 1006-13.

4. Handerson J. Respiratory monitoring. In: Miller's anesthesia. 7th ed. Edited by Miller RD: Philadelphia, Elsvier ChurchillLivingstone. 2010, p 1601.

5. Cohen SP, Anderson PL. Mucoid impaction following nasal intubation in a child with an upper respiratory infection. J Clin Anesth 1998; 10: $327-30$. 